\title{
Thrombocytopenia - a challenge for Pediatric Emergency Departments
}

\author{
Daniela Pop ${ }^{1}$, Helen Emilsson², Daniela Dreghiciư ${ }^{3}$, Lucia Cinezan ${ }^{3}$ \\ ${ }^{1}$ University of Medicine and Pharmacy "Iuliu Hatieganu” Cluj-Napoca, Romania \\ ${ }^{2}$ Lund University Hospital, Lund, Sweden \\ ${ }^{3}$ Emergency Department, Emergency Hospital for Children, Cluj-Napoca, Romania
}

\begin{abstract}
Objectives. This study aimed to find the most frequent causes of thrombocytopenia in patients who come to the Pediatric Emergency Department and to evaluate the importance of the investigations and immediate treatment options available in the Emergency Department for the final diagnosis.

Methods. The study included 73 pediatric patients (aged two months to 16 years) with thrombocytopenia evaluated in the Pediatric Emergency Department in 6 years. The final diagnosis of the patients presenting with thrombocytopenia in the Pediatric Emergency Department was recorded. We assessed the clinical characteristics and findings of the investigations of the patients diagnosed with immune thrombocytopenic purpura (ITP). Results. Most of the patients were diagnosed with sepsis (40\%). The second most frequent diagnosis was ITP (34\%). Values of the platelets recorded in patients with ITP were between 1,000 and 87,000 and 14/25 (56\%) of the patients with values lower than $20,000 / \mathrm{mm} 3$. Most of the patients with ITP received i.v. immunoglobulin. Conclusions. ITP and sepsis were the leading causes of thrombocytopenia in children in the Pediatric Emergency Department in our hospital. The suspicion of malignant hematological disorders was raised based on the data from the patient's medical history and the findings in the physical exam. Treatment with i.v. immunoglobulin in patients with ITP should only be used when patients associate active hemorrhage. The conclusions of this study allowed us to establish a protocol for the investigation and treatment of patients with ITP.
\end{abstract}

Keywords: thrombocytopenia, children, immune thrombocytopenic purpura, immunoglobulin

\begin{abstract}
Abbreviations
CRP: C reactive protein

ESR: erythrocyte sedimentation rate

ITP: immune thrombocytopenic purpura

i.v.: intravenous

SD: standard deviation
\end{abstract}

\section{INTRODUCTION}

One of the problems that pediatricians or emergency medicine doctors have to face in the Emergency Departments or on-call rooms is the evaluation and treatment of patients with thrombocytopenia. The mechanisms leading to thrombocytopenia are decreased production (in bone marrow failure syndromes), increased destruction of the platelets, platelet sequestration and hemodilution. In some diseases, multiple mechanisms can contribute to this condition (1). The rea- sons for which children with thrombocytopenia are brought in the Emergency Department are diverse, depending on the cause that leads to this; it can sometimes be the first manifestation of severe disease. Most of the times the diagnosis is precise, but there are situations in which there is the need for more investigations, that are not available 24 hours a day or 7 days a week, to confirm or rule out, for instance, a malignant hematological disease or an autoimmune disease.

Many times patients with the first manifestations of ITP are being brought in the Emergency 
Department. ITP is a condition characterized by isolated thrombocytopenia (a platelet count of less than $100,000 / \mu 1$ ), a consequence of the autoimmune destruction of normal platelets, at a rate that exceeds production by the bone marrow (2). It is a condition which is diagnosed anywhere around the world mainly on the exclusion of other diseases that could explain the thrombocytopenia. There are problems of differential diagnosis, establishing a treatment plan and making a decision regarding admitting or sending the patient at home, with recommendations, with or without treatment.

\section{OBJECTIVES}

This study aimed to find the most frequent causes of thrombocytopenia in pediatric patients who come to the Emergency Department of the Emergency Hospital for Children in Cluj-Napoca. Another purpose was to evaluate the clinical characteristics and results of the investigations of patients with ITP. Finally, we wanted to see what the best available treatment options for our patients are and establish a local protocol for the management of patients with thrombocytopenia.

\section{MATERIAL AND METHODS}

The study took place between 01.01.2010 and 01.01 .2016 and included 73 children with thrombocytopenia, meaning a platelet count of less than $100,000 / \mu l$. The study included patients who were brought to the Emergency Department of the Emergency Hospital for Children in Cluj-Napoca, either by the parents or sent from other hospitals. It was a retrospective, descriptive study with data collected from the patient's charts. The data collected included: the age and sex of the patient, clinical manifestations, investigations (complete blood count, CRP, ESR, procalcitonin, liver enzymes, urea, creatinine, coagulation tests, abdominal ultrasound) partial or final diagnosis and treatment.

\section{RESULTS}

There were 73 patients with thrombocytopenia evaluated in our Emergency Department in 6 years
(Table1). Most of them were diagnosed with sepsis $(40 \%)$. The second most frequent diagnosis was ITP. Only newly diagnosed patients with ITP were included in the study. There were also 5 patients diagnosed with hemolytic uremic syndrome and 4 patients who were eventually diagnosed as having leukemia. Other causes of thrombocytopenia were: hypersplenism in a patient with cirrhosis, systemic lupus erythematosus, liver failure in a patient with mushroom poisoning etc.

TABLE 1. Diagnosis of the patients with thrombocytopenia, mean age of patients and mean values $\pm S D$ of the platelets in the Emergency Department

\begin{tabular}{|l|c|c|c|}
\hline Diagnosis & $\begin{array}{c}\text { Number of } \\
\text { patients (\%) }\end{array}$ & $\begin{array}{c}\text { Mean age } \\
\pm \text { SD }\end{array}$ & $\begin{array}{c}\text { Value of the } \\
\text { platelets (mean } \\
\text { value } \pm \text { SD) }\end{array}$ \\
\hline ITP & $25 / 73(34.2 \%)$ & $4.9 \pm 3.5$ years & $18,050 \pm 21,811 / \mu \mathrm{l}$ \\
\hline Sepsis & $29 / 73(39.7 \%)$ & $2.3 \pm 3$ years & $46,137 \pm 29,372 / \mu \mathrm{l}$ \\
\hline $\begin{array}{l}\text { Hemolytic } \\
\text { uremic } \\
\text { syndrome }\end{array}$ & $5 / 73(6.8 \%)$ & $1.43 \pm 1.7$ years & $69,200 \pm 18,211 / \mu \mathrm{l}$ \\
\hline Leukemia & $4 / 73(5.4 \%)$ & $8 \pm 6.1$ years & $35,666 \pm 22,479 / \mu \mathrm{l}$ \\
\hline $\begin{array}{l}\text { Other } \\
\text { diseases }\end{array}$ & $10 / 73(13.6 \%)$ & & \\
\hline
\end{tabular}

There were 25 children with ITP, aged between 2 months and 16 years, 16 boys (64\%). The clinical characteristics of the patients with ITP are summarized in Table 2. Values of the platelets recorded in these patients were between 1,000 and $87,000 / \mu 1$ and $14 / 25(56 \%)$ of the patients with values lower than $20,000 / \mu 1$. Four patients with ITP associated anemia, with values of the hemoglobin between 9.8 and $10.8 \mathrm{~g} / \mathrm{dl}$.

Treatment with i.v. immunoglobulin $\mathrm{G}$ was administered in 19 cases $(76 \%)$ in the Emergency Department. In 6 of the cases of ITP, sent to us from other hospitals, treatment with corticosteroids was started in the hospital they came from.

\section{DISCUSSIONS}

We started this study from the idea that we frequently see in the Emergency Department patients with severe thrombocytopenia, the most significant challenges being the cases in which the cause cannot be precisely determined, but the correct treatment must be started as soon as

TABLE 2. Clinical characteristics and management of the patients with ITP in the Emergency Department

\begin{tabular}{|c|c|c|c|c|c|c|c|c|}
\hline \multicolumn{7}{|c|}{ Clinical data } & \multicolumn{2}{|l|}{ Treatment } \\
\hline Purpura & Echimosis & Epistaxis & $\begin{array}{l}\text { Gum } \\
\text { bleeding }\end{array}$ & Hematuria & Splenomegaly & $\begin{array}{l}\text { Head/face } \\
\text { trauma }\end{array}$ & $\begin{array}{l}\text { i.v } \\
\text { Immunoglobulin } \\
\end{array}$ & $\begin{array}{l}\text { No treatment in the } \\
\text { Emergency Department }\end{array}$ \\
\hline $\begin{array}{l}23 / 25 \\
(92 \%)\end{array}$ & $10 / 25(40 \%)$ & $5 / 25(20 \%)$ & $3 / 25(12 \%)$ & $2 / 25(8 \%)$ & $3 / 35(12 \%)$ & 2 & $19 / 25(76 \%)$ & $6 / 25(24 \%)$ \\
\hline
\end{tabular}


possible. The purpose of this study was to find the most frequent causes of thrombocytopenia in children brought directly to the Emergency Department of the Emergency Hospital for Children in Cluj-Napoca. We also aimed to establish a protocol for the diagnosis and management of these patients, given the fact that many of the investigations necessary to make a final diagnosis in patients with thrombocytopenia are not available 24 hours/day or during the weekend. On the other hand, some studies might be essential to make before treatment is started, as some treatment measures might impair the results of future investigations.

A thorough medical history (family medical history, previous diseases, recent viral infections, medications or vaccinations, bleedings, onset of the symptoms etc.) and physical exam (parameters of the vital signs, general condition, lymphadenopathies, hepatomegaly, splenomegaly, distribution of the purpura, skin color, mucosal bleedings etc.) remain the most important aspects in the evaluation and decisions related to management (investigations, treatment, hospitalization, reference to a pediatric hematologist) of the patients with thrombocytopenia.

Most of the patients were diagnosed with sepsis. Among the patients diagnosed with sepsis, 6 were previously diagnosed with acute leukemia (one with myeloblastic and 5 with lymphoblastic leukemia), so the mechanisms for the thrombocytopenia were multiple in these cases. There were 6 patients with sepsis and meningoencephalitis and 2 patients with fulminant purpura. The medical history and physical exam, as well as the investigations, routinely performed in the emergency laboratory, allowed in most of these cases a definite diagnosis.

In 5 of the patients diagnosed with ITP the viral agent who triggered the antiplatelet antibody production: Varicella-zoster virus in 3 cases, EpsteinBarr virus in one patient and CMV in one case.

In newly diagnosed patients with ITP there is always the question of excluding a malignant hematologic disorder, a task which is difficult to achieve in an Emergency Department, because neither peripheral blood smear nor bone marrow aspiration or biopsy can be performed 24 hours a day or during the weekend, a pediatric hematologist not being available all the time. So, general pediatricians and specialists in emergency medicine are faced with the challenge of carefully considering the proper treatment for patients with ITP.

There is no specific test to confirm this diagnosis, so in the Pediatric Emergency Department, an algorithm for the differential diagnosis of thrombocytopenia is needed. On the other hand, a proper exclusion or confirmation of some diseases (like malignant hematological disorders, autoimmune diseases etc.) is not possible. Previous long term studies showed that about $14 \%$ of children initially diagnosed with ITP were eventually diagnosed with other disorders like familial thrombocytopenia, systemic lupus erythematosus, Wiskott Aldrich syndrome etc. (3) or associated diseases $(4,5)$.

A relatively small number of patients with ITP associated significant bleedings of the mucosa in our study. Two of the patients also had a facial or cranial trauma; these circumstances represent risk factors for intracranial bleeding. There was no patient in our study with this severe and potentially life- threatening complication.

The guidelines for ITP state that most of the patients don't need admittance in the hospital. It is imperative though to properly inform the parents about the risks of severe illness and that in case of bleeding the child should be reevaluated. It is a difficult task to achieve in an Emergency Department where the number of cases seen in 24 hours is usually very high. Another important aspect is to make sure that a pediatrician or a pediatric hematologist follows the patient's evolution. Parents need to make sure that the child will avoid any contact sports, trauma and drugs that could accentuate thrombocytopenia. So sometimes to make sure that these indications are followed we prefer keeping the patients in the hospital. Guidelines recommend admitting patients with ITP in case of active bleeding, platelets number under $20,000 / \mu 1$ and if the parent can't follow the indications or they live far from the hospital, and the access to a medical unit is difficult.

In a recent study which also evaluates the reasons of admission of the patients with ITP assessed in the emergency departments, Jones et al. notice that the percentages are variable in different hospitals (between 20 and 100\%), observing that bleedings and their severity were not the main criteria for admittance (6). The authors mention a variety of factors that contribute to the decision of admittance, like distance to the hospital, the possibilities of the family to come back to the hospital, the level of activity of the patient and the risk of being exposed to trauma, the anxiety of the patient and parents, the expectations of the parents from the pediatrician (6). All of our patients with ITP were referred to the pediatric hematologist for observation, further investigations, treatment, and follow-up. 
The lowest values of the platelets were recorded in patients with ITP, $11 / 25$ patients presenting values lower than $10,000 / \mathrm{mm}^{3}$. Most of the patients without bleeding or mild bleedings (cutaneous bleeding) don't need treatment (2) regardless of the number of platelets. i.v. immunoglobulin in one dose or on a short term is recommended as first-line therapy in case of active bleeding. i.v. immunoglobulin should be used when a rapid increase of the platelets is needed. There is no evidence to support the long term use of corticosteroid therapy versus the short term use of corticosteroids. An important issue regarding the fact that many patients (76\%) with ITP received treatment with i.v. immunoglobulin is related to the costs. It is a problem raised by other studies too, in close relation to the issue of disseminating and implementing guidelines $(7,8)$.

Choosing the right therapy in children with ITP in the Emergency Department is a difficult task. Recent international guidelines recommend in newly diagnosed patients with ITP a conservative approach, based on observing the patient, at home, avoiding trauma and starting treatment only in active hemorrhages $(1,2)$.

Warrier R. and Chauhan A. state, in a review about ITP, that "the factors that influence the selection of a treatment regime in a given patient are quality of life impact, adverse events, the likelihood of response, bleeding risk, patient/parent anxiety, and economic issues" (9). Recent recommendations regarding the treatment of patients with ITP state that patients with no bleeding or mild bleeding (only skin manifestations like petechiae or ecchymosis) can be managed with only observation without any treatment, regardless of the platelet count $(10,11)$. It is a difficult decision to make in the Emergency Department.

In our study, most of the patients received i.v. immunoglobulin $\mathrm{G}$ a treatment which has the ad- vantage of a quick response in the number of platelets, does not interfere with the results of the bone marrow aspiration or biopsy, but is a costly treatment. Recent guidelines recommend administration of i.v. immunoglobulin $\mathrm{G}$ only in cases which present bleedings of the mucosa $(2,12)$.

The results of this study have to be seen in light of some limitations. First, it is a retrospective study, where the data were collected from the patients' files. A second factor that affects the accuracy of the findings is linked to the difficulty of follow-up, patients being sent to other hospitals, after the initial evaluation and treatment. Third, doctors who manage these patients in the Pediatric Emergency Department are specialists in pediatrics and emergency medicine, the experience in pediatric oncohematology being limited.

The interpretation of the results of this study, the review of the guidelines, surveys, and experiences of other centers led to the elaboration of a protocol adapted for the specific conditions of our Emergency Department. Following this study, we reduced significantly the number of cases in which we decide to administer i.v. immunoglobulin G, limiting this treatment option in the Emergency Department to those patients who have moderate or severe bleeding.

\section{CONCLUSIONS}

ITP and sepsis were the leading causes of thrombocytopenia in children in the Pediatric Emergency Department in our hospital. The suspicion of malignant hematological disorders was raised based on the data from the patient's medical history and the findings in the physical exam. Treatment with i.v. immunoglobulin in patients with ITP should only be used when patients associate active hemorrhage.

Conflict of interest: none declared Financial support: none declared

\section{REFERENCES}

1. Labarque V, Van Geet C. Clinical practice: Immune thrombocytopenia in paediatrics. Eur J Pediatr 2014; 173: 163-172.

2. Neunert C, Lim W, Crowther M et al. The American Society of Hematology 2011 evidence-based practice guideline for immune thrombocytopenia. Blood 2011; 117 (16): 4190-207.

3. Bryant N, Watts $R$. Thrombocytopenic syndromes masquerading as childhood immune thrombocytopenic purpura. Clin Pediatr 2011; 50 (3): 225-230.

4. Lee AC. Isolated thrombocytopenia in childhood: What if it is not immune thrombocytopenia? Singapore Med J 2018; 59 (7): 390-393

5. Neunert CE. Management of newly diagnosed immune thrombocytopenia: Can we change outcomes? Blood Adv 2017; 1(24): 2295-2301.

6. Jones L, Koch T, Stanek J, O'Brien SH. Patterns of Emergency Department Care for Newly Diagnosed Immune Thrombocytopenia in United States Children's Hospitals. J Pediatr 2017; 190: 265-267.

7. Witmer CM, Lambert MP, O'Brien SH et al. Multicenter Cohort Study Comparing U.S. Management of Inpatient Pediatric Immune Thrombocytopenia to Current Treatment Guidelines. Pediatr Blood Cancer 2016; 63:1227-1231.

8. Schultz CL, Mitra N, Schapira MM et al. Influence of the American Society of Hematology Guidelines on the Management of Newly Diagnosed Childhood Immune Thrombocytopenia. JAMA Pediatr 2014; 168 (10): e142214.

9. Warrier R, Chauhan A. Management of Immune Thrombocytopenic Purpura: An Update. Ochsner J 2012; 12 (3): 221-227. 
10. Grimaldi-Bensouda L, Nordon C, Leblanc T et al. Childhood immune thrombocytopenia: A nationwide cohort study on condition management and outcomes. Pediatr Blood Cancer 2017; 64 (7): 1-8.

11. Okubo $\mathrm{Y}$, Michihata N, Morisaki N et al. Recent trends in practice patterns and comparisons between immunoglobulin and corticosteroid in pediatric immune thrombocytopenia. Int J Hematol 2018; 107 (1): 75-82.

12. Jang JH, Kim JY, Mun YC et al. Management of immune thrombocytopenia: Korean experts recommendation in 2017. Blood Res 2017; 52 (4): 254-263. 